\title{
Microwave Spectrum, Conformational Equilibria, Intramolecular Hydrogen Bonding and Centrifugal Distortion of 3-Butene-1-thiol
}

\author{
K.-M. Marstokk and Harald Møllendal ${ }^{\star}$
}

Department of Chemistry, The University of Oslo, P.O.Box 1033, Blindern, N-0315, Oslo 3, Norway

\begin{abstract}
Marstokk, K.-M. and Møllendal, Harald, 1986. Microwave Spectrum, Conformational Equilibria, Intramolecular Hydrogen Bonding and Centrifugal Distortion of 3-Butene-1-thiol. - Acta Chem. Scand. A 40: 402-411.

The microwave spectra of 3-butene-1-thiol, $\mathrm{HSCH}_{2} \mathrm{CH}_{2} \mathrm{CH}=\mathrm{CH}_{2}$, and one deuterated species, $\mathrm{DSCH}_{2} \mathrm{CH}_{2} \mathrm{CH}=\mathrm{CH}_{2}$, have been investigated at about $-60^{\circ} \mathrm{C}$. Three conformations of the molecule were assigned. The heavy atom gauche conformation has an intramolecular hydrogen bond formed between the mercapto group hydrogen atom and the $\pi$ electrons of the double bond. The two others which were assigned, were denoted Extended I and Extended II. They have no $\mathrm{H}$ bonds. The internal energy difference between Extended $I$ and the H-bonded gauche conformation is $2.9(5) \mathrm{kJ} \mathrm{mol}^{-1}$, the latter being more stable. The $\mathrm{H}$ bonded gauche conformation is, furthermore, $3.6(6) \mathrm{kJ} \mathrm{mol}^{-1}$ more stable than Extended II.

Several excited states were assigned for all three rotamers, and some vibrational frequencies of the gauche form were determined by relative intensity measurements. Extensive centrifugal distortion analyses were carried out on the $\mathrm{H}$ bonded gauche conformer.
\end{abstract}

The conformational properties of 3-buten-1-ol, $\mathrm{HOCH}_{2} \mathrm{CH}_{2} \mathrm{CH}=\mathrm{CH}_{2}$ have been studied by infrared $^{1.2}$ and microwave ${ }^{3}$ (MW) spectroscopic methods as well as by electron diffraction. ${ }^{4}$ Both in solution ${ }^{1.2}$ and in the gas phase,$^{3,4}$ the most stable conformation of this compound has an intramolecular hydrogen bond ( $\mathrm{H}$ bond) formed between the hydroxyl group hydrogen atom and the $\pi$ electrons of the double bond. It was estimated ${ }^{3}$ that this rotamer is at least $3 \mathrm{~kJ} \mathrm{~mol}^{-1}$ more stable than any other form in the free state. Moreover, the hydrogen bond apparently modifies the $\mathrm{C} 1$ C2-C3-C4 dihedral angle ( $\alpha$ in Fig. 1). Instead of the normal $\alpha=60^{\circ}$, this angle is $75(3)^{\circ}$ in 3 buten-1-ol, ${ }^{3}$ bringing the $\pi$ electrons into closer proximity with the hydroxyl group hydrogen atom allowing a stronger $\mathrm{H}$ bond to be formed. ${ }^{3}$

3-Butene-1-thiol, $\mathrm{HSCH}_{2} \mathrm{CH}_{2} \mathrm{CH}=\mathrm{CH}_{2}$, was chosen for study in order to compare the ability of the mercapto and the hydroxyl groups to form internal $\mathrm{H}$ bonds with $\pi$ electrons. In general, the hydroxyl group is able to form stronger hydrogen

\footnotetext{
*To whom correspondence should be addressed.
}

bonds than the mercapto group under similar conditions. This was confirmed in our study. The weaker internal hydrogen bond of the thiol manifests itself in two different ways. In addition to the more stable hydrogen-bonded conformation shown in Fig. 2, two non-hydrogen-bonded $e x$ tended structures, also shown in the same figure, were sufficiently populated to be assigned, unlike the case of $\mathrm{HOCH}_{2} \mathrm{CH}_{2} \mathrm{CH}=\mathrm{CH}_{2} \cdot{ }^{3,4}$ In the more stable hydrogen-bonded conformation of $\mathrm{HSCH}_{2} \mathrm{CH}_{2} \mathrm{CH}=\mathrm{CH}_{2}$, the C1-C2-C3-C4 dihedral angle has the normal value of $57(3)^{\circ}$ in contrast to the $75(3)^{\circ}$ of the corresponding alcohol. ${ }^{3}$

\section{Experimental}

$\mathrm{HSCH}_{2} \mathrm{CH}_{2} \mathrm{CH}=\mathrm{CH}_{2}$ was synthesized largely as described in the literature, ${ }^{5,6}$ purified by gas chromatography, and identified by IR, PMR and ${ }^{13} \mathrm{C}$ NMR spectroscopy. Extensive microwave spectroscopic measurements were made in the 26.5$38.0 \mathrm{GHz}$ spectral region. Selected measurements were also made in the $K$ band. The conventional Stark modulated MW spectrometer 


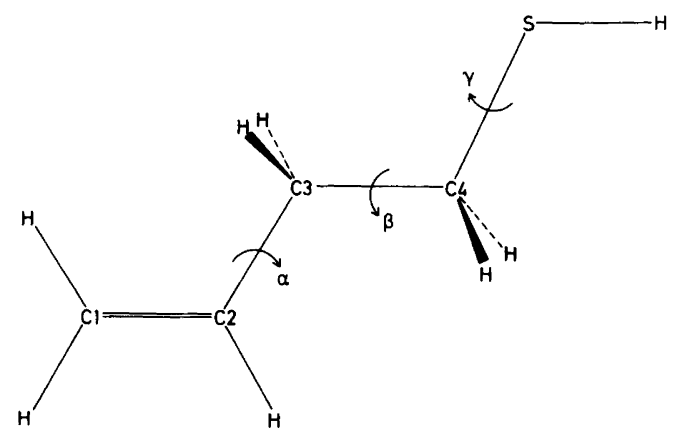

Fig. 1. Atom numbering and dihedral angles $\alpha=$ $\angle \mathrm{C} 1 \mathrm{C}_{2} \mathrm{C} 3 \mathrm{C} 4, \beta=\angle \mathrm{C} 2 \mathrm{C} 3 \mathrm{C} 4 \mathrm{~S}$, and $\gamma=\angle \mathrm{C} 3 \mathrm{C} 4 \mathrm{SH}$. The conformation shown has $\alpha=\beta=\gamma=0^{\circ}$. Rotation around $\alpha$ by $57^{\circ}, \beta$ by $115^{\circ}$, and $\gamma$ by $130^{\circ}$ in the direction indicated by the arrows produces the hydrogen-bonded gauche conformation. Similarly, $\alpha$ $=62^{\circ}, \beta=2^{\circ}$, and $\gamma=120^{\circ}$ produces Extended $l$, while $\alpha=62^{\circ}, \beta=5^{\circ}$, and $\gamma=240^{\circ}$ yields Extended II.

used has been briefly described before. ${ }^{7}$ The cell was cooled with dry ice to about $-60^{\circ} \mathrm{C}$. Lower temperatures could not be used due to insufficient vapour pressure of the compound. A vapour pressure of about 1-2 Pa was employed during the spectral measurements. The deuterated species, $\mathrm{DSCH}_{2} \mathrm{CH}_{2} \mathrm{CH}=\mathrm{CH}_{2}$, was produced by direct exchange with heavy water in the wave guide.

\section{Results}

Microwave spectrum. The microwave spectrum of $\mathrm{HSCH}_{2} \mathrm{CH}_{2} \mathrm{CH}=\mathrm{CH}_{2}$ was quite dense at approximately $-60^{\circ} \mathrm{C}$. The strongest lines of the spectrum, $a$ type $R$ branch as well as $b$ type $Q$ branch transitions of the hydrogen-bonded gauche conformation (Fig. 2), were scattered all over the spectrum. The strongest of these transitions had peak absorption coefficients of about $4 \times 10^{-7} \mathrm{~cm}^{-1}$ near $-60^{\circ} \mathrm{C}$. Another prominent feature of the spectrum was the lumps of lines which occured every $2.8 \mathrm{GHz}$. They were modulated at low Stark voltages and assigned as the $a$ type $R$ branch pile-ups of the two highly prolate extended conformations depicted in Fig. 2.

Assignment of the hydrogen-bonded conformation. $\mathrm{HSCH}_{2} \mathrm{CH}_{2} \mathrm{CH}=\mathrm{CH}_{2}$ has three single bonds, rotation about which gives rise to a number of conformations. In Fig. 1, the correspond- ing dihedral angles are designated $\alpha, \beta$, and $\gamma$, respectively, and assigned the value of $0^{\circ}$. Rotation of $\alpha \approx 60^{\circ}, \beta \approx 120^{\circ}$, and $\gamma \approx 120^{\circ}$ in the directions indicated by the arrows of this figure produces approximately the hydrogen-bonded conformation (see also Fig. 2). Bond moment calculations ${ }^{8}$ predicted $\mu_{a} \approx 1 \mathrm{D}, \mu_{b} \approx 0.8 \mathrm{D}$, and $\mu_{c} \approx 0.5 \mathrm{D}$, for this conformation; therefore, search was initially made for the $a$ type $R$ branch transitions. The $J=8 \leftarrow 7$ and $J=9 \leftarrow 8 a$ type series were found first; $b$ type $Q$ and $R$ branch transitions were then readily identified. A total of about 130 lines were measured with $J \leq 31$. A portion of the spectrum is listed in Table 1 and the resulting spectroscopic constants ( $I$ representation, $A$ reduction $^{9}$ ) are listed in Table 2. The complete spectra are available from the authors upon request or from the Molecular Spectra Data Center, Molecular Spectroscopy Division, National Bureau of Standards, B268 Physics Building, Gaithersburg, MD 20899, USA, where they have been deposited.

High $J, P$ and $R$ branch, $b$ type transitions were not sufficiently strong to be identified with certainty. The frequencies of relatively strong $c$ type $Q$ branch lines could be very accurately predicted. However, none of these transitions were seen in the spectrum presumably because $\mu_{c}$ was too small to produce sufficient intensities. The dipole moment was of interest, but the low $J$ lines were too weak to allow quantitative Stark effect measurements to be made. Hence, no dipole moment could be determined. The deuterated species, $\mathrm{DSCH}_{2} \mathrm{CH}_{2} \mathrm{CH}=\mathrm{CH}_{2}$ was studied in order to locate unambiguously the mercapto group hydrogen atom position. The assignment of this<smiles>CC1C=CC2C=CC1CCC2</smiles>

H-BONDED GAUCHE<smiles>C=CC1C2CC3CC(C2)C(S)C1C3</smiles>

EXTENDED 1<smiles>C=CC1CC2CCC1C(S)C2</smiles>

EXTENDED II
Fig. 2. Newman projections of the three conformations assigned in this work viewed along the C3-C4 bond. The H-bonded gauche conformation is more stable than Extended / by $2.9(5) \mathrm{kJ} \mathrm{mol}^{-1}$; and more stable than Extended I/ by $3.6(6) \mathrm{kJ} \mathrm{mol}^{-1}$. 
MARSTOKK AND MØLLENDAL

Table 1. Selected ground state transitions of the hydrogen-bonded gauche conformation of $\mathrm{HSCH}_{2} \mathrm{CH}_{2} \mathrm{CH}=\mathrm{CH}_{2}$.

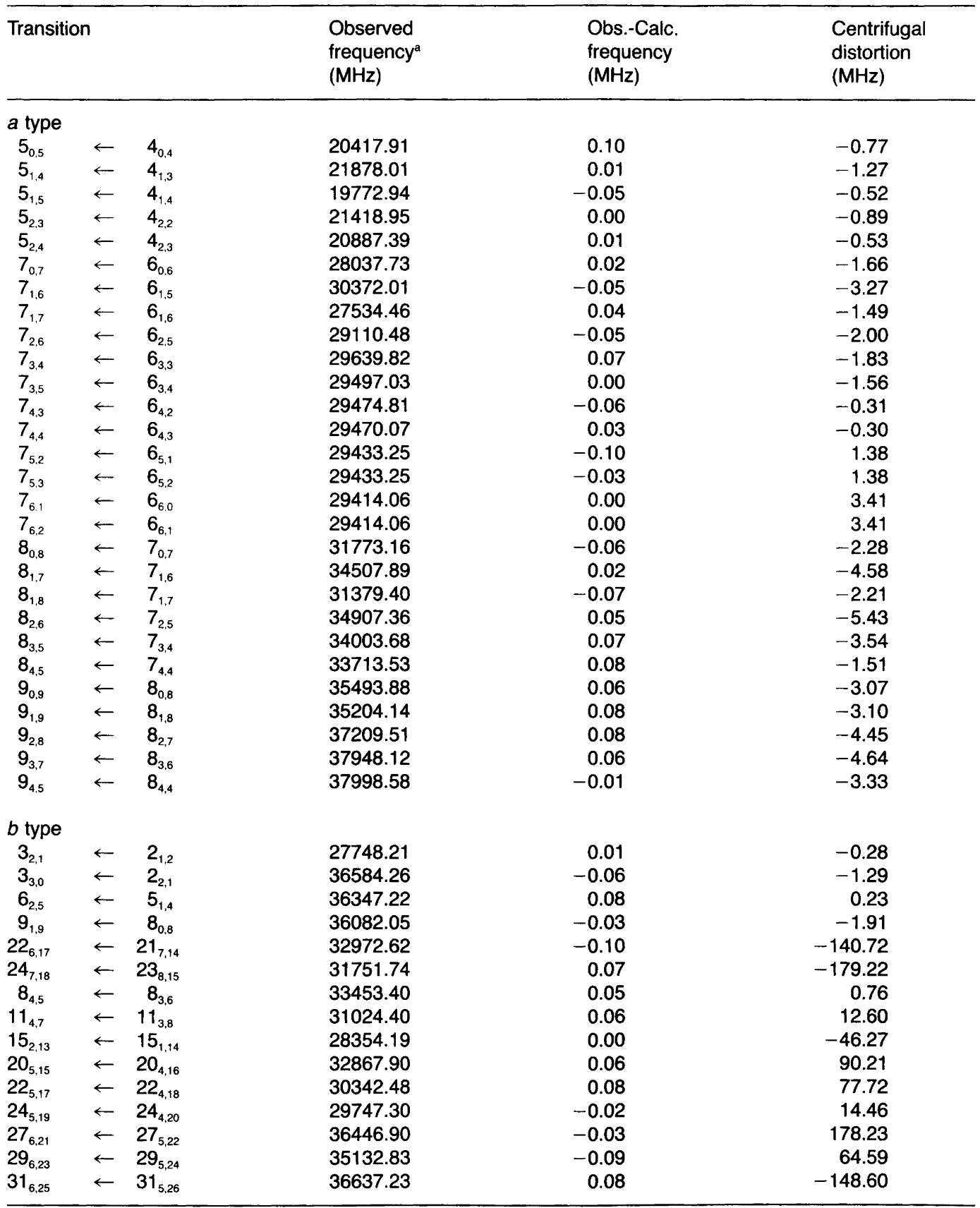

a $\pm 0.10 \mathrm{MHz}$. 
Table 2. Spectroscopic constants ${ }^{a}$ of the ground vibrational states of the hydrogen-bonded gauche conformation of $\mathrm{HSCH}_{2} \mathrm{CH}_{2} \mathrm{CH}=\mathrm{CH}_{2}$ and $\mathrm{DSCH}_{2} \mathrm{CH}_{2} \mathrm{CH}=\mathrm{CH}_{2}$.

\begin{tabular}{lcc}
\hline $\begin{array}{l}\text { Species } \\
\text { No. of }\end{array}$ & $\mathrm{HSCH}_{2} \mathrm{CH}_{2} \mathrm{CH}=\mathrm{CH}_{2}$ & $\mathrm{DSCH}_{2} \mathrm{CH}_{2} \mathrm{CH}=\mathrm{CH}_{2}$ \\
transitions & 117 & 78 \\
$\begin{array}{l}\text { Root-mean- } \\
\text { square dev./ } \\
\mathrm{MHz}\end{array}$ & 0.065 & 0.072 \\
\hline
\end{tabular}

$\begin{array}{lcc}A_{0} / \mathrm{MHz} & 6894.4387(34) & 6703.6341(87) \\ B_{0} / \mathrm{MHz} & 2308.11700(84) & 2302.6352(41) \\ C_{0} / \mathrm{MHz} & 1882.05016(78) & 1868.0054(41) \\ \Delta_{J} / \mathrm{kHz} & 2.2710(26) & 2.118(30) \\ \Delta_{J K} / \mathrm{kHz} & -13.346(14) & -12.478(29) \\ \Delta_{K} / \mathrm{kHz} & 33.22(13) & 28.69(39) \\ \Delta_{j} / \mathrm{kHz} & 0.70608(68) & 0.7076(11) \\ \Delta_{K} / \mathrm{kHz} & 3.885(25) & 3.747(37)\end{array}$

${ }^{a}$ Uncertainties represent one standard deviation.

spectrum was straightforward. The resulting spectroscopic constants are shown in Table 2 . Kraitchman's coordinates ${ }^{10}$ for the mercapto group hydrogen atom are listed in Table 8 .

Vibrationally excited states. The ground state lines were accompanied by several excited state transitions. Four of these, belonging to three different normal modes, were assigned as shown in Table 3. The strongest of these excited state transitions were about $55 \%$ as strong as the corre- sponding ground state lines. Relative intensity measurements made largely as prescribed in Ref. 11 yielded $85(20) \mathrm{cm}^{-1}$ for what was assumed to be the first excited state of the torsion around the $\mathrm{C} 2-\mathrm{C} 3$ bond. This is close to $93(4) \mathrm{cm}^{-1}$ determined for the corresponding frequency of skew 1butene, ${ }^{12}$ and $84(20) \mathrm{cm}^{-1}$ found for 3-buten-1ol. ${ }^{3}$ The second excited state of this vibration was also assigned. It is seen from Tables 2 and 3 that the successive changes of the rotational constants upon excitation are fairly constant. This is typical for a harmonic mode. The same result was also found for 3-buten-1-ol ${ }^{3}$ for the corresponding vibration.

The second strongest satellite series was assumed to belong to the first excited state of the torsional vibration around the $\mathrm{C} 3-\mathrm{C} 4$ bond. Its intensity was approximately $40 \%$ of that of the ground state at about $-60^{\circ} \mathrm{C}$. Relative intensity measurements ${ }^{11}$ yielded $137(30) \mathrm{cm}^{-1}$, somewhat less than $172(15) \mathrm{cm}^{-1}$ found for the corresponding normal mode of 3-buten-1-ol. ${ }^{3}$ The final excited state (Table 3 ), assigned to the lowest bending mode, had a relative intensity of about $30 \%$ of that of the ground state, which yielded 181(40) $\mathrm{cm}^{-1}$. The relatively large changes of the rotational constants upon excitation (Tables 2 and 3) are typical for a mode involving heavy atoms, as a low frequency vibration is presumed to do.

No assignments could be made for the C-S torsion despite many attempts. This vibration was estimated to have a frequency of about $200 \mathrm{~cm}^{-1}$ (Ref. 13).

Table 3. Spectroscopic constants ${ }^{a}$ of vibrationally excited states of the hydrogen-bonded gauche conformation of $\mathrm{HSCH}_{2} \mathrm{CH}_{2} \mathrm{CH}=\mathrm{CH}_{2}$.

\begin{tabular}{|c|c|c|c|c|}
\hline $\begin{array}{l}\text { Vibr. state } \\
\text { N.o.t. }{ }^{b} \\
\text { R.m.s. }{ }^{c} \mathrm{MHz}\end{array}$ & $\begin{array}{c}\text { First ex. } \\
\text { C2C3 tors. } \\
88 \\
0.084\end{array}$ & $\begin{array}{c}\text { Second ex. } \\
\text { C2C3 tors. } \\
66 \\
0.098\end{array}$ & $\begin{array}{c}\text { First ex. } \\
\text { C3C4 tors. } \\
50 \\
0.100\end{array}$ & $\begin{array}{l}\text { First ex. lowest } \\
\text { bend. vib. } \\
33 \\
0.096\end{array}$ \\
\hline$A_{\sqrt{v}} / \mathrm{MHz}$ & $6953.8912(90)$ & $7014.340(14)$ & $6924.208(17)$ & $6913.947(22)$ \\
\hline$B \sqrt{ } / \mathrm{MHz}$ & $2298.9670(45)$ & $2289.5159(60)$ & $2299.3234(83)$ & $2296.1561(98)$ \\
\hline$C_{\sqrt{ }} / \mathrm{MHz}$ & $1876.7078(45)$ & $1871.2330(62)$ & 1876.7423(84) & $1876.7218(97)$ \\
\hline$\Delta, / \mathrm{kHz}$ & $2.302(34)$ & $2.073(45)$ & $1.968(59)$ & $2.123(67)$ \\
\hline$\Delta_{J K} / \mathrm{kHz}$ & $-13.170(31)$ & $-13.393(47)$ & $-13.747(42)$ & $-12.796(68)$ \\
\hline$\Delta_{K} / \mathrm{kHz}$ & $34.77(45)$ & $39.28(66)$ & $33.00(65)$ & $32.6(10)$ \\
\hline$\Delta_{j} / \mathrm{kHz}$ & $0.6712(11)$ & $0.6384(15)$ & $0.7176(15)$ & $0.6921(21)$ \\
\hline$\Delta_{k} / \mathrm{kHz}$ & $3.845(41)$ & $4.004(61)$ & $3.868(54)$ & $3.327(87)$ \\
\hline
\end{tabular}

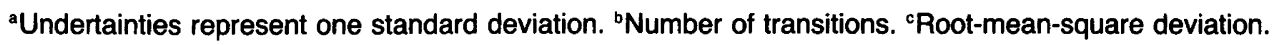


MARSTOKK AND MØLLENDAL

Table 4. Approximate ${ }^{a}$ values of $B+C$ of the ground and prominent excited states of Extended I.

\begin{tabular}{|c|c|c|}
\hline $\begin{array}{l}\text { Species } \\
\text { Vibrational } \\
\text { state }\end{array}$ & $\begin{array}{c}\frac{\mathrm{HSCH}_{2} \mathrm{CH}_{2} \mathrm{CH}=\mathrm{CH}_{2}}{\approx(B+C) / \mathrm{MHz}} \\
\end{array}$ & $\begin{array}{c}\mathrm{DSCH}_{2} \mathrm{CH}_{2} \mathrm{CH}=\mathrm{CH}_{2} \\
\approx(B+C) / \mathrm{MHz}\end{array}$ \\
\hline Low bending ${ }^{b}$ & 2831.0 & $--^{c}$ \\
\hline Ground & 2833.1 & 2795.5 \\
\hline C3-C4 or C-S torsion ${ }^{\mathrm{b}}$ & 2833.5 & 2795.9 \\
\hline Bending or torsion ${ }^{b}$ & 2837.2 & 2797.6 \\
\hline First ex. C2-C3 torsion ${ }^{\mathrm{b}}$ & 2837.8 & 2800.6 \\
\hline Second ex. C2-C3 torsion & 2842.5 & 2805.9 \\
\hline
\end{tabular}

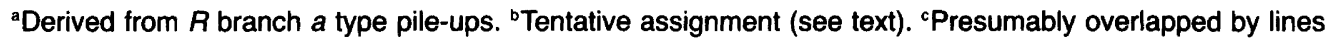
from Extended II.

Assignment of Extended I and Extended II. The $\alpha$ and $\beta$ angles defined in Fig. 1 have the values $\alpha \approx 60^{\circ}$ and $\beta \approx 0^{\circ}$ for all three possible extended conformations. The $\gamma$ angle is $120^{\circ}$ for Extended $I$, and $240^{\circ}$ for Extended II (see Fig. 1). The $\gamma$ angle may also take the value $0^{\circ}$, but this extended conformation was not found, as will be discussed below.

Both Extended I and Extended II are nearly symmetrical tops, both with the asymmetry parameter $^{14} x \approx-0.999$. The principal axes components of the dipole moment, predicted using the bond moment method, ${ }^{8}$ were $\mu_{a} \approx 1.4 \mathrm{D}$, $\mu_{b} \approx 0.1 \mathrm{D}$, and $\mu_{c} \approx 0.4 \mathrm{D}$ for Extended $I$, and $\mu_{a} \approx 1.2 \mathrm{D}, \mu_{b} \approx 0.5 \mathrm{D}$, and $\mu_{c} \approx 0.5 \mathrm{D}$ for $E x$ tended II. Typical $a$ type $R$ branch pile-ups appearing almost exactly each $B+C$ were thus expected for these two conformations. The pile-ups referred to above, which occurred each $2.8 \mathrm{GHz}$ throughout the MW spectrum, were assigned to these two forms. Extended $I$ and $I I$ had only marginally different values of $B+C$. In addition, both of them have several low lying vibrations which are well populated at about $-60^{\circ} \mathrm{C}$. This gave rise to a complicated fine structure of the pile-ups. An unique assignment of the ground states of Extended I and II could be made only by studying the deuterated species.

The changes of $B+C$ upon deuteration were calculated to be about $-36 \mathrm{MHz}$ for Extended I, and approximately $-42 \mathrm{MHz}$ for Extended $\mathrm{II}$. The strongest pile-up of the parent species had $B+C \approx 2833.1 \mathrm{MHz}$ whereas the strongest pile-up of the deuterated species had $B+C \approx$ $2795.5 \mathrm{MHz}$ (see Table 4 ). The $\Delta(B+C$ ) was thus $-37.6 \mathrm{MHz}$, and, for this reason, the strongest pile-ups were assigned to Extended $I$. It was also possible to assign the rather weak low $K_{-1}$ transitions of this conformation, as shown in Table 5. The resulting spectroscopic constants $\left(I^{r}\right.$ representation, $A$ reduction ${ }^{9}$ ) are listed in Table 6. Unfortunately, it was not possible to make similar assignments for the deuterated species due to insufficient intensities. Several pile-ups arising from vibrationally excited states of $E x$ tended $I$ were also assigned as shown in Table 4 using the shifts upon deuteration as a criterion.

Extended II was then assigned in the same manner as Extended I (see Table 7). The changes of $B+C$ upon deuteration were found to be $-44.8 \mathrm{MHz}$, as inferred from Table 7. It was not possible to make a definite assignment of low $K_{-1}$ transitions of this conformation. Hence, no complete set of rotational constants could be determined because of the complicated nature of the pile-up spectral regions, and because the intensities of the Extended II transitions were roughly $50 \%$ of the corresponding transitions of Extended $I$. However, a tentative set of $A_{0}=$ 29745.(735) $\mathrm{MHz}, B_{0}=1429.315(54) \mathrm{MHz}$, and $C_{0}=1408.769(52) \mathrm{MHz}$ (not reported in Table 7) with a root-mean-square deviation of $0.23 \mathrm{MHz}$ was derived from 12 transitions. Two excited state pile-up series were definitely assigned for this conformation as seen in Table 7. Semiquantitative intensity measurements, as well as probable spectral shifts of the excited state pile-ups were used to make the tentative assignments for the excited states as presented in Tables 4 and 7 . In addition to the $\approx B+C$ values shown in Tables 
Table 5. Ground state transitions of the Extended / conformation of $\mathrm{HSCH}_{2} \mathrm{CH}_{2} \mathrm{CH}=\mathrm{CH}_{2}$.

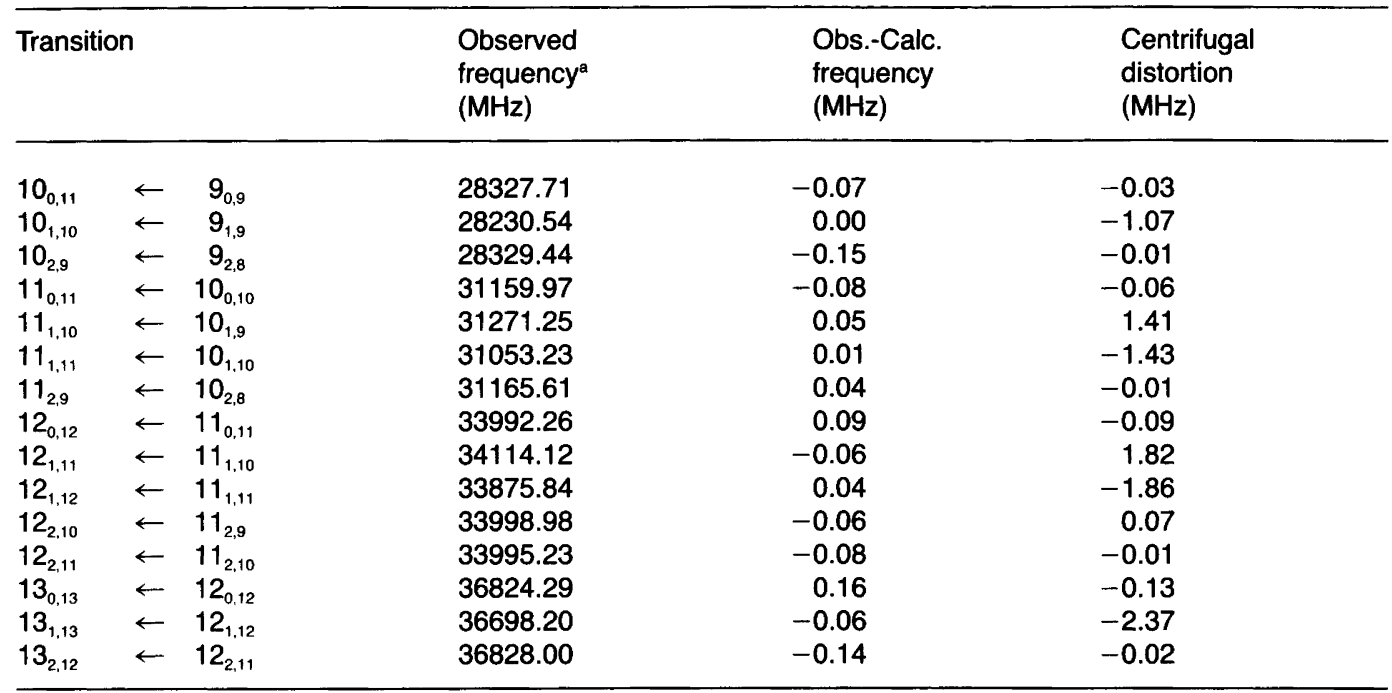

${ }^{\mathrm{a}} \pm 0.15 \mathrm{MHz}$.

4 and 7 , several additional weaker pile-up series were seen, but it was not possible to assign them uniquely to either Extended I, or to Extended II.

Table 6. Spectroscopic constants ${ }^{\mathrm{a}}$ of the ground vibrational state of the Extended / conformation of $\mathrm{HSCH}_{2} \mathrm{CH}_{2} \mathrm{CH}=\mathrm{CH}_{2}$.

\begin{tabular}{lr}
\hline$A_{0} / \mathrm{MHz}$ & $23944(383)$ \\
$B_{0} / \mathrm{MHz}$ & $1426.275(28)$ \\
$C_{0} / \mathrm{MHz}$ & $1406.716(28)$ \\
$\delta_{J} / \mathrm{kHz}^{\mathrm{b}}$ & $-0.267(51)$ \\
\hline
\end{tabular}

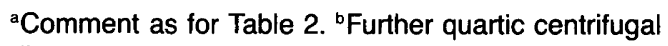
distortion constants preset at zero.
It was noted above that the extended conformation with $\gamma=0^{\circ}$ was not identified. This form too, was predicted to have $x \approx-0.999$. Its dipole moment components were predicted ${ }^{8}$ to be roughly $\mu_{a} \approx 0.8 \mathrm{D}, \mu_{b} \approx 1.1 \mathrm{D}$, and $\mu c \approx 0.3 \mathrm{D}$; moreover, $\Delta(B+C) \approx-65 \mathrm{MHz}$ upon deuteration. No shifts from the crowded pile-up regions compatible with $\Delta(B+C) \approx-65 \mathrm{MHz}$ were seen. Therefore, this conformer remains unassigned. However, this rotamer corresponds to the anti conformation of $\mathrm{CH}_{3} \mathrm{CH}_{2} \mathrm{SH}$, which is less stable than the gauche form of ethanethiol ${ }^{15}$ by $1.5 \mathrm{~kJ} \mathrm{~mol}^{-1}$. The gauche conformation of $\mathrm{CH}_{3} \mathrm{CH}_{2} \mathrm{SH}$ corresponds both to Extended $I$ and II. Due to the lower $\mu_{a}$ and the higher energy expected for the extended conformation with $\gamma=0^{\circ}$,

Table 7. Approximate ${ }^{a}$ values of $B+C$ of the ground and prominent excited states of Extended II.

\begin{tabular}{lcc}
\hline $\begin{array}{l}\text { Species } \\
\text { Vibrational } \\
\text { state }\end{array}$ & $\mathrm{HSCH}_{2} \mathrm{CH}_{2} \mathrm{CH}=\mathrm{CH}_{2}$ & $\frac{\mathrm{DSCH}_{2} \mathrm{CH}_{2} \mathrm{CH}^{2} \mathrm{CH}_{2}}{$\cline { 3 - 3 }} \\
\hline Ground & $\approx(B+C) / \mathrm{MHz}$ & $\approx(B+C) / \mathrm{MHz}$ \\
C3-C4 torsion or C-S torsion $^{\mathrm{b}}$ & 2838.2 & 2793.4 \\
First ex. C2-C3 torsion $^{b}$ & 2838.4 & 2793.6 \\
\hline
\end{tabular}

${ }^{\mathrm{a}, \mathrm{b}}$ Comments as for Table 4. 
MARSTOKK AND MØLLENDAL

Table 8. Plausible molecular structure ${ }^{\text {a }}$ (bond lengths $/ \mathrm{pm}$; angles $/{ }^{\circ}$ ) of the three conformations assigned for $\mathrm{HSCH}_{2} \mathrm{CH}_{2} \mathrm{CH}=\mathrm{CH}_{2}$.

Structural parameters kept fixed

\begin{tabular}{llll}
\hline$C=C$ & 133.1 & $\angle C-S-H$ & 96.0 \\
$C 2-C 3$ & 149.6 & $\angle C-C-S$ & 113.6 \\
$C 3-C 4$ & 152.8 & $\angle C 2 C 3 C 4$ & 111.6 \\
$C-S$ & 181.4 & $\angle C 1 C 2 C 3$ & 127.8 \\
$S-H$ & 133.6 & $\angle C 3 C 2 H$ & 115.5 \\
$C 3-H$ & 109.3 & $\angle H C 1 C 2$ & 121.5 \\
$C 4-H$ & 109.3 & $\angle C 3 C 4 H$ & 109.47 \\
$C 1-H$ & 109.0 & $\angle C 4 C 3 H$ & 109.47 \\
$C 2-H$ & 109.0 & $\angle H C 3 H$ & 109.47 \\
& & $\angle H C 4 H$ & 109.47 \\
& & $\gamma^{\mathrm{b}}$ & 120.0 Extended I \\
& & & 240.0 Extended II \\
\hline
\end{tabular}

Fitted structural parameters

\begin{tabular}{lccc}
\hline Conformation & H-bonded gauche & Extended I & Extended II \\
\hline$\alpha(\angle \mathrm{C} 1 \mathrm{C} 2 \mathrm{C} 3 \mathrm{C} 4)^{\mathrm{b}}$ & $57(3)$ & $62(3)$ & $62(3)$ \\
$\beta(\angle \mathrm{SC} 4 \mathrm{C} 3 \mathrm{C} 2)^{\mathrm{b}}$ & $115(3)(65(3)$ from syn $)$ & $2(3)$ & $5(3)$ \\
$\gamma(\angle \mathrm{HSC} 4 \mathrm{C} 3)^{\mathrm{b}}$ & $130(5)$ & $-^{\mathrm{c}}$ & $-^{\mathrm{c}}$ \\
\hline
\end{tabular}

Rotational constants for H-bonded gauche conformation

\begin{tabular}{|c|c|c|c|c|c|c|}
\hline \multirow[t]{2}{*}{ Species } & \multicolumn{3}{|c|}{$\mathrm{HSCH}_{2} \mathrm{CH}_{2} \mathrm{CH}=\mathrm{CH}_{2}$} & \multicolumn{3}{|c|}{$\mathrm{DSCH}_{2} \mathrm{CH}_{2} \mathrm{CH}=\mathrm{CH}_{2}$} \\
\hline & Obs. & Obs.-Calc. & Diff.(\%) & Obs. & Obs.-Calc. & Diff.(\%) \\
\hline $\mathrm{A} / \mathrm{MHz}$ & 6894.44 & -58.88 & 0.85 & 6703.63 & -70.30 & 1.05 \\
\hline $\mathrm{B} / \mathrm{MHz}$ & 2308.12 & -10.08 & 0.44 & 2302.64 & -9.60 & 0.41 \\
\hline $\mathrm{C} / \mathrm{MHz}$ & 1882.05 & -9.82 & 0.52 & 1868.01 & -9.17 & 0.49 \\
\hline
\end{tabular}

Kraitchman's coordinates ${ }^{10}$ of the mercapto group hydrogen atom of H-bonded conformation

\begin{tabular}{llll}
\hline & \multicolumn{1}{c}{$|\mathrm{a}|$} & \multicolumn{1}{c}{$|\mathrm{b}|$} & \multicolumn{1}{c}{$|\mathrm{c}|$} \\
\hline From rotational constants & $0.47425(38)$ & $1.33914(14)$ & $\begin{array}{l}0.55400(34) \\
\text { From plausible structure }\end{array}$ \\
\hline
\end{tabular}

Hydrogen-bond parameters

\begin{tabular}{lrlr}
\hline $\mathrm{H} \cdots \mathrm{C} 2$ & 260 & $\mathrm{H} \cdots \mathrm{C} 1$ & 297 \\
$\mathrm{~S} \cdots \mathrm{C} 2$ & 326 & $\mathrm{~S} \cdots \mathrm{C} 1$ & 389 \\
$\angle \mathrm{S}-\mathrm{H} \cdots \mathrm{C} 1$ & 124 & $\angle \mathrm{S}-\mathrm{H} \cdots \mathrm{C} 2$ & 107 \\
$\angle \mathrm{H} \cdots \mathrm{C} 2=\mathrm{C} 1$ & 93 & $\angle \mathrm{H} \cdots \mathrm{C} 1=\mathrm{C} 2$ & 61 \\
$\angle \mathrm{S}-\mathrm{H}, \mathrm{C} 1=\mathrm{C} 2^{\text {d }}$ & 47 & $\angle \mathrm{C}-\mathrm{S}, \mathrm{C} 1=\mathrm{C} 2^{\ominus}$ & 63 \\
\hline
\end{tabular}




\begin{tabular}{|c|c|c|c|}
\hline $\mathrm{H} \cdots \mathrm{C}^{9}$ & 290 & $S \cdots C^{9}$ & 355 \\
\hline \multicolumn{4}{|c|}{ Rotational constants for Extended I } \\
\hline \multirow[t]{2}{*}{ Species } & \multicolumn{3}{|c|}{$\mathrm{HSCH}_{2} \mathrm{CH}_{2} \mathrm{CH}=\mathrm{CH}_{2}$} \\
\hline & Obs. & Obs.-Calc. & Diff.(\%) \\
\hline $\begin{array}{l}\mathrm{A} / \mathrm{MHz} \\
\mathrm{B} / \mathrm{MHz} \\
\mathrm{C} / \mathrm{MHz}\end{array}$ & $\begin{array}{c}23944(383)^{n} \\
1426.28 \\
1406.72\end{array}$ & $\begin{array}{l}- \\
-2.45 \\
-0.18\end{array}$ & $\begin{array}{r}-\overline{17} \\
0.01\end{array}$ \\
\hline \multirow[t]{2}{*}{ Species } & \multicolumn{3}{|c|}{$\mathrm{DSCH}_{2} \mathrm{CH}_{2} \mathrm{CH}=\mathrm{CH}_{2}$} \\
\hline & Obs. & Obs.-Calc. & Diff.(\%) \\
\hline$\approx(B+C) / \mathrm{MHz}$ & 2795.5 & -4.32 & 0.15 \\
\hline
\end{tabular}

Rotational constants for Extended II

\begin{tabular}{|c|c|c|c|c|c|c|c|}
\hline \multirow[t]{2}{*}{ Species } & \multirow[b]{2}{*}{ - } & \multicolumn{3}{|c|}{$\mathrm{HSCH}_{2} \mathrm{CH}_{2} \mathrm{CH}=\mathrm{CH}_{2}$} & \multicolumn{3}{|c|}{$\mathrm{DSCH}_{2} \mathrm{CH}_{2} \mathrm{CH}=\mathrm{CH}_{2}$} \\
\hline & & Obs. & Obs.-Calc. & Diff.(\%) & Obs. & Obs.-Calc. & Diff.(\%) \\
\hline$\approx(B+C) / \mathrm{MHz}$ & & 2838.2 & -0.5 & 0.02 & 2793.4 & -0.3 & 0.01 \\
\hline
\end{tabular}

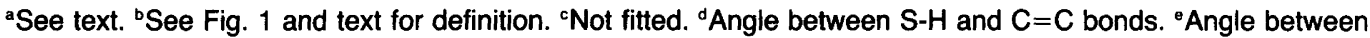
$\mathrm{C}-\mathrm{S}$ and $\mathrm{C}=\mathrm{C}$ bonds. 'Ref. 18 . ${ }^{9}$ van der Waals radius of carbon assumed to be $170 \mathrm{pm}$ as in aromatic molecules. ${ }^{18}$ hToo inaccurate to be used for fitting.

as compared to the other two extended conformations, it is not surprising that this rotamer was not identified in the rather dense spectrum.

Energy difference. Unfortunately, it was not possible to determine the dipole moments of any of the three rotamers assigned in this work. The dipole moment components which are crucial for the spectral intensities were predicted using the bond-moment method. ${ }^{8}$ The values given above were employed in the internal energy difference computations. The $K_{-1}=1$ lines of Extended $I$ were compared with transitions from the hydrogen-bonded gauche conformation. Harrington's technique of power-saturating the transitions ${ }^{16}$ as well as the standard use of completely unsaturated lines ${ }^{17}$ were employed. The statistical weights of both rotamers were assumed to be the same, i.e. 2. In this manner, the hydrogen- bonded gauche conformation was found to be 2.9 $\mathrm{kJ} \mathrm{mol}^{-1}$ more stable than Extended I. One standard deviation was liberally estimated to be \pm 0.5 $\mathrm{kJ} \mathrm{mol}{ }^{-1}$, taking the uncertainties of the dipole moment components into consideration. The energy difference between Extended $I$ and Extended II was then found by comparing the pile-up intensities in the limit of no power saturation. ${ }^{17}$ Both extended rotamers were assumed to have the same statistical weight. Extended I was found to be $0.7(2) \mathrm{kJ} \mathrm{mol}^{-1}$ more stable than $E x$ tended II. The uncertainty was estimated to be one standard deviation. The hydrogen-bonded gauche conformation was thus $3.6(6) \mathrm{kJ} \mathrm{mol}^{-1}$ more stable than Extended II.

Searches for further conformations. The assignments reported above included all strong lines of the MW spectrum, as well as the majority of tran- 
sitions of intermediate intensities and many weak lines. It is very likely that any conceivable conformation of $\mathrm{HSCH}_{2} \mathrm{CH}_{2} \mathrm{CH}=\mathrm{CH}_{2}$ would have a sizable dipole moment. From the fact that there were no relatively strong lines left unassigned, we concluded that the three rotamers assigned in this work were also the three most stable rotamers of 3-butene-1-thiol, and it was conservatively estimated that the hydrogen-bonded gauche conformer was at least $3 \mathrm{~kJ} \mathrm{~mol}^{-1}$ more stable than any other possible unassigned rotamers.

Structure. The two investigated isotopic species furnished insufficient information for a complete structural determination. Assumptions had to be made in order to derive important structural parameters. The $\mathrm{C} 1 \mathrm{C} 2 \mathrm{C} 3 \mathrm{C} 4$ dihedral angle $(\alpha$ of Fig. 1) and the SC4C3C2 dihedral angle ( $\beta$ of Fig. 1) were fitted for all three rotamers. In addition, the HSC4C3 dihedral angle ( $\gamma$ of Fig. 1) was fitted for the $\mathrm{H}$-bonded gauche conformation. The rest of the structural parameters shown in Table 8 were taken from recent, accurate studies of closely related compounds. The dihedral angles were chosen for fitting because the rotational constants are very sensitive to their variation and because they are chemically interesting. The dihedral angles were fitted in steps of $1^{\circ}$. The results are shown in Table 8; the uncertainties quoted are error limits of approximately three standard deviations. The $\alpha$ angles are close to $60^{\circ}$ for all three conformations, as compared to $75(3)^{\circ}$ found for the H-bonded 3-buten-1-ol. ${ }^{3}$ The $\beta$ angles are close to the values expected for completely staggered rotamers. This was also found for 3-buten-1-ol. ${ }^{3}$ The increase of the $\gamma$ angle of the $\mathrm{H}$-bonded gauche conformation to $130(5)^{\circ}$ from the usual $120^{\circ}$ leads to a shorter distance between the mercapto group hydrogen atom and the $\mathrm{C} 1$ and $\mathrm{C} 2$ carbon atoms.

Kraitchman's coordinates ${ }^{10}$ of the mercapto group hydrogen atom of the $\mathrm{H}$-bonded form are also shown in Table 8 . They are in reasonable agreement with the values calculated from the plausible molecular structure, and show beyond doubt that this hydrogen atom is oriented in the most favourable way for intramolecular hydrogen bonding interaction.

\section{Discussion}

There is considerable evidence that a weak intramolecular hydrogen bond is formed in the gauche conformation. The fact that this rotamer actually has the mercapto group hydrogen atom directed in the most favourable orientation for this kind of interaction is one piece of evidence. Moreover, the $\mathrm{H} \cdots \mathrm{C} 2$ distance of $260 \mathrm{pm}$ (Table 8 ), is somewhat shorter than the sum of the van der Waals radii of hydrogen and aromatic carbon ${ }^{18}$ (i.e. 290 $p m$ ). The $\mathrm{S} \cdots \mathrm{C} 2$ distance is also shorter than the sum of the van der Waals radii ${ }^{18}$ by about $30 \mathrm{pm}$. The $\gamma$ angle of $130(5)^{\circ}$ instead of $120^{\circ}$ is also indicative. The fact that the gauche rotamer is considerably more stable than any other conformation is further evidence. This would be hard to explain if one had to exclude a stabilizing interaction between the mercapto group and the double bond.

The major conformational difference between the H-bonded gauche and the Extended I rotamer involves only the rotation about the $\mathrm{C} 3-\mathrm{C} 4$ bond and no further relaxations. It has been argued (see Ref. 19 for a discussion) that the internal hydrogen bond strength in such a case is approximately equal to the internal energy difference between the two rotamers involved. The hydrogen bond strength is thus about $2.9(5) \mathrm{kJ} \mathrm{mol}^{-1}$ found for the internal energy difference between the hydrogen-bonded gauche rotamer and Extended I. The stronger hydrogen bond in the most stable form of $\mathrm{HOCH}_{2} \mathrm{CH}_{2} \mathrm{CH}=\mathrm{CH}_{2}{ }^{3}$, as compared to $\mathrm{HSCH}_{2} \mathrm{CH}_{2} \mathrm{CH}=\mathrm{CH}_{2}$, presumably has consequences both for the $\alpha$ angles of the conformers in the two cases, $75(3)^{\circ}$ vs. $57(3)^{\circ}$, and the structural composition, as mentioned in the introduction. The stronger hydrogen bond of the alcohol is supposed to increase the energy difference between its $\mathrm{H}$-bonded gauche and extended conformations. The populations of the extended rotamers thus become sufficiently small as to render them undetectable by MW spectroscopy. ${ }^{3}$

Acknowledgement. Mr. Marko Opresnik is thanked for synthesizing the sample used in this work. 


\section{References}

1. Oki, M. and Iwamura, H. Bull. Chem. Soc. Jpn. 32 (1959) 567.

2. Ditter, W. and Luck, A. P. Ber. Bunsenges. Phys. Chem. 75 (1971) 163.

3. Marstokk, K.-M. and Møllendal, H. Acta Chem. Scand. A 35 (1981) 395.

4. Trætteberg, M. and Østensen, H. Acta Chem. Scand. A33 (1979) 491.

5. Surzur, J.-M., Crozet, M.-P. and Dupuy, C. C.R. Hebt. Seances Acad. Sci., Paris, Series C, 264 (1967) 610.

6. Walling, C. and Pearson, M. S., J. Am. Chem. Soc. 86 (1964) 2262.

7. Marstokk, K.-M. and Møllendal, H. J. Mol. Struct. 5 (1970) 205.

8. Smyth, C. P. Dielectric Behavior and Structure, McGraw-Hill, New York 1955, p. 244.

9. Gordy, W. and Cook, R. L. Microwave Molecular Spectra, Wiley, New York 1984, p. 324.

10. Kraitchman, J. Am. J. Physics 21 (1953) 17.
11. Esbitt, A. S. and Wilson, E. B. Jr. Rev. Sci. Instrum. 34 (1963) 901.

12. Kondo, S., Hirota, E. and Morino, Y. J. Mol. Spectrosc. 28 (1968) 471.

13. Inagaki, F., Harada, I. and Shimanouchi, T. $J$. Mol. Spectrosc. 46 (1973) 381.

14. Gordy, W. and Cook, R. L. Microwave Molecular Spectra, Wiley, New York 1984, p. 229.

15. Schmidt, R. E. and Quade, C. R. J. Chem. Phys. 62 (1975) 3864.

16. a. Harrington, H. W. J. Chem. Phys. 46 (1967) 3698; b. Ibid., 49 (1968) 3023.

17. Townes, C. H. and Schawlow, A. L. Microwave Spectroscopy, McGraw-Hill, New York 1955, p. 372.

18. Pauling, L. The Nature of the Chemical Bond, 3rd ed., Cornell University Press, New York 1960, p. 260.

19. Marstokk, K.-M. and Møllendal, H. Acta Chem. Scand. A39 (1985) 15.

Received February 7, 1986. 